\title{
les micromécanismes de déformations plastiques dans un sol granulaire
}

\author{
B. CAMBOU \\ Professeur, Ecole Centrale de Lyon*
}

\section{Résumé:}

L'analyse de la distribution orientée des forces de contact dans un milieu granulaire simple a permis d'identifier et de préciser deux mécanismes d'écrouissage, l'un lié à l'évolution des valeurs moyennes des forces de contact, l'autre à l'évolution de la largeur des distributions de ces forces. Ces résultats sont replacés dans le cadre d'une analyse élastoplastique. Nous montrons que le modèle ainsi défini permet de traduire l'essentiel des comportements observés expérimentalement dans les sols granulaires.

\section{Abstract:}

The analysis of the orientational distributions of contact forces in a granular material is allowed to identify and define two hardening mecanisms, the first one is related with the evolution of the mean contact forces and the second is related with the evolution of the wideness of the distributions of these forces. These results are considered in the frame of an elastoplastic approach. Then it is shown that the proposed model allow to describe a good lot of experimentally observed beheaviors of granular materials.

* Laboratoire de Mécanique des Solides, 36, route de Dardilly, BP 17, 69130 Ecully. 


\section{INTRODUCTION}

A ce jour la modélisation du comportement des matériaux granulaires n'est pas satisfaisante en particulier dans le cas de sollicitations complexes.

L'une des raisons de ce relatif échec tient à ce que, pour ce type de matériau, l'analyse des micromécanismes de déformations irréversibles est toujours restée extrêmement sommaire, contrairement à ce qui a été fait par exemple pour les métaux. Pour ces matériaux, l'approche élasto-plastique, nourrie par une analyse des micro-phénomènes, proposée par les métallurgistes, s'est avérée très satisfaisante.

Le but de cet article est donc de proposer une analyse statique d'un milieu granulaire simple afin d'éclairer certains mécanismes de déformations irréversibles ainsi que les variables fondamentales commandant ces mécanismes [9].

Nous nous bornerons à analyser un milieu granulaire bidimensionnel formé de cylindres égaux dans un assemblage quelconque non régulier.

\section{L'APPROCHE MICROSCOPIQUE}

Le micromécanisme fondamental de déformation irréversible dans un milieu granulaire est le glissement entre grains.

La condition de glissement au contact (fig. 1) s'écrit:

$\left|T^{k}\right|=\mu N^{k}$

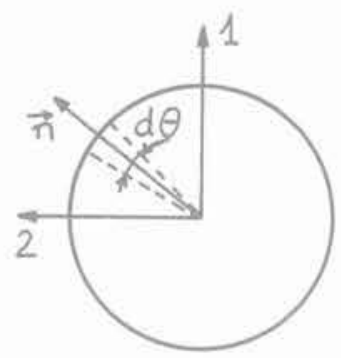

Fig. 1 .

$\mu$ étant le coefficient de frottement du matériau constituant les particules.

La relation (1) qui régit l'existence des déformations irréversibles dans le milieu ne fait intervenir que les forces de contact, l'analyse statique parait donc a priori la plus adaptée pour décrire ces déformations.

La variable statique à grande échelle (macro) est le tenseur des contraintes noté $\sigma_{\alpha \beta}$. Les variables statiques à l'échelle des grains (micro) sont les forces de contact, dont les composantes sont notées $\mathrm{F}_{\alpha}^{\mathrm{k}}$

L'orientation des points de contact caractérise la géométrie de l'assemblage de la structure granulaire, c'est donc un paramètre essentiel.
L'ensemble des $F_{\alpha}^{k}$ est décomposé en sous-ensembles définis pour une orientation donnée (n) qui seront notés $\mathrm{F}_{\alpha}^{\prime}(\overrightarrow{\mathrm{n}})$. Soit $\mathrm{P}(\overrightarrow{\mathrm{n}})$ la probabilité d'existence d'un contact d'orientation compris entre les limites $\theta \pm$ $\mathrm{d} \theta / 2$ (fig. 2).

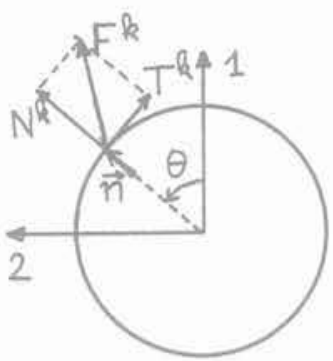

Fig. 2.

$\mathrm{N}$ étant le nombre de contact par unité de volume dans un volume $V$ le nombre de ces contacts est égal à:

$$
\operatorname{NVP}(\vec{n}) \mathrm{d} \theta
$$

Le volume $\mathrm{V}$ analysé est supposé suffisamment grand pour que ce nombre soit grand.

Les sous-ensembles $F_{0}^{i}(\vec{n})$ sont formés d'un nombre important d'éléments discrets qui peuvent être décrits par une distribution (fig. 3).

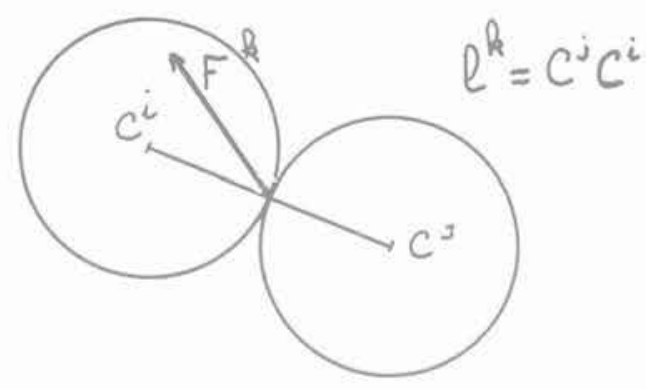

Fig. 3. - Distribution des variables $F_{\alpha}$.

Ces distributions sont définies de façon approchée par leurs moments statistiques.

La précision de la description augmente avec le nombre de moments pris en compte.

Nous allons analyser tout d'abord une description très simplifiée limitée au premier moment statistique (la valeur movenne) puis nous proposerons une description à partir des deux premiers moments (moyenne et écart type).

\section{DESCRIPTION STATISTIQUE \\ AU PREMIER ORDRE (MÉCANISME D'ÉCROUISSAGE N 1)}

Les variables microstatiques sont décrites par la valeur moyenne notée $\overrightarrow{F_{\alpha}(\vec{n})}$ des sous-ensemble $F_{\alpha}^{k}(\vec{n})$. 


\subsection{Relations entre les variables micro et macrostatiques.}

La relation permettant de calculer la variable macrostatique $\left(\sigma_{\alpha \beta}\right)$ à partir des variables microstatiques $\left(\mathrm{F}_{\alpha}^{\mathrm{k}}\right)$ a été établi par Weber [15]:

$$
\sigma_{\alpha \beta}=\frac{1}{V} F_{\alpha}^{k} l_{\beta}^{k}
$$

$V$ est le volume analysé et la sommation sur $k$ est étendue à tous les contacts dans le volume $\mathrm{V}$ (fig. 4).

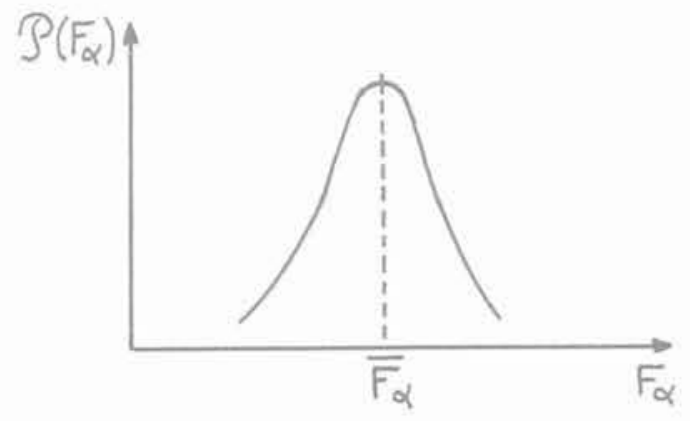

Fig. 4.

Pour un matériau formé de cylindres de diamètre $\mathrm{D}$ la relation (2) s'écrit $[2,6]$

$$
\sigma_{\alpha \beta}=N D \int_{0}^{\pi} P(\vec{n}) \overline{F_{\alpha}(\vec{n})} n_{\beta} d \theta
$$

Cette relation fait apparaître le produit $\mathrm{P}(\overrightarrow{\mathrm{n}}) \overline{\mathrm{F}_{\alpha}(\overrightarrow{\mathrm{n}})}$ dont il paraît difficile de dissocier les deux termes. C'est donc ce produit que nous avons choisi comme variable microstatique. On remarquera que ce produit associe une variable statique $\overline{\mathrm{F}_{\alpha}(\bar{n})}$ et une variable caractérisant la géométrie de la structure $\mathrm{P}(\overrightarrow{\mathrm{n}})$.

Nous cherchons à définir la variable microstatique $\left(\mathrm{P}(\overrightarrow{\mathrm{n}}) \mathrm{F}_{\mathrm{\alpha}}(\overrightarrow{\mathrm{n}})\right)$ à partir de la variable macrostatique $\left(\sigma_{\alpha \beta}\right)$.

La relation (3) exprimée dans le repère principal de $\sigma_{\alpha \beta}$ nous conduit à proposer l'expression de $\mathrm{P}(\overrightarrow{\mathrm{n}}) \mathrm{F}_{\Delta}(\vec{n})$ sous la forme [4]:

$$
P(\vec{n}) \overline{F_{\Delta}(\vec{n})}=N D \sigma_{\Delta} f_{\Delta}(\vec{n})
$$

L'emploi de l'indice grec majuscule $\Delta$ signifie que cette relation n'est valable que dans le repère principal de $\sigma_{\alpha \beta}$, et sans sommation sur l'indice $\Delta$.

La relation $(5)$ exprime que $P(\vec{n}) \overline{F_{\Delta}(\vec{n})}$ est le produit d'un terme $\sigma_{A}$ qui caractérise la sollicitation et d'un terme $\mathrm{NDf}_{\Delta}(\vec{n})$ qui caractérise l'état interne de la structure granulaire.

Si l'on reporte (5) dans (3), on constate que $f_{\alpha}(\vec{n})$ doit satisfaire aux conditions suivantes:

$$
\int_{0}^{\pi} f_{\Delta} n_{\Gamma} d \theta=\delta_{\Delta \Gamma}
$$

On notera que $f_{\alpha}(\vec{n})$ est une fonction vectorielle que l'on pourra exprimer aisément dans un repère quelconque à partir de $\mathrm{f}_{\Delta}(\hat{\mathrm{n}})$.

Pour une sollicitation isotrope appliquée à une structure isotrope, la symétrie du milieu impose que: $f_{\alpha}^{(0)}(\mathrm{n})$ $=A n_{\alpha}$

compte tenu de (6) on aura donc:

$$
\mathrm{f}_{\alpha}^{(0)}(\vec{n})=\frac{2}{\pi} \mathrm{n}_{\alpha}
$$

L'état caractérisé par $\mathrm{f}_{\alpha}^{(0)}(\overrightarrow{\mathrm{n}})$ sera défini comme «l'état vierge :.

\subsection{Discrêtisation de l'état interne}

La relation (5) définit la fonction $\mathrm{f}_{\alpha}(\overrightarrow{\mathrm{n}})$ qui caractérise l'état interne du milieu.

Nous cherchons à exprimer cette fonction à partir d'un nombre fini de paramètres. Pour cela, nous introduisons un développement de $f_{\alpha}(\vec{n})$ en fonction de $\vec{n}[1,5]$ :

$$
\mathrm{f}_{\alpha}(\overrightarrow{\mathrm{n}})=\psi_{\alpha \beta} \mathrm{n}_{\beta}+\psi_{\alpha \beta \gamma \delta} \mathrm{n}_{\beta} \mathrm{n}_{\gamma} \mathrm{n}_{\delta}
$$

Nous avons montré [6] que pour un matériau bidimensionnel ce développement s'écrit

$\frac{\pi}{2} f_{1}(\vec{n})=n_{1}+\lambda_{11}\left(n_{1}^{2}-3 n_{2}^{2}\right) n_{1}+\lambda_{12}$

$\left(\mathrm{n}_{2}^{2}-3 \mathrm{n}_{1}^{2}\right) \mathrm{n}_{2}$

$\frac{\pi}{2} f_{2}(\vec{n})=n_{2}+\lambda_{21}\left(n_{1}^{2}-3 n_{2}^{2}\right) n_{1}+\lambda_{22}$

$\left(n_{2}^{2}-3 n_{1}^{2}\right) n_{2}$

Les quatre variables $\lambda_{\alpha \beta}$ caractérisent l'état interne du milieu. Pour un milieu isotrope soumis à une sollicitation à directions principales fixes $\lambda_{12}=\lambda_{21}=0$

Dans ce cas, l'état du milieu ne dépend que de 2 variables internes $\lambda_{11}$ et $\lambda_{22}$.

Dans la suite de cette étude, nous nous limiterons à ce type de sollicitations.

\subsection{Signification physique des variables d'état}

Pour une sollicitation donnée, les variables d'état $\lambda_{\alpha \beta}$ caractérisent la distribution du vecteur $\mathrm{P}(\overrightarrow{\mathrm{n}}) \mathrm{F}_{\alpha}(\overrightarrow{\mathrm{n}})$, en particulier on notera sur la figure 5 que l'orientation $\gamma$ de ce vecteur par rapport à $\vec{n}$ est très sensible à la valeur des $\lambda_{\text {ap. }}$.

Considérons la décomposition suivante des contraintes principales $\sigma_{\Delta}$ :

$$
\sigma_{\Delta}=\sigma_{\Delta}^{(n)}+\sigma_{\Delta}^{(t)}
$$

$\sigma_{\Delta}{ }^{(n)}$ est la part de $\sigma_{\Delta}$ due uniquement aux composantes normales des forces de contact. 

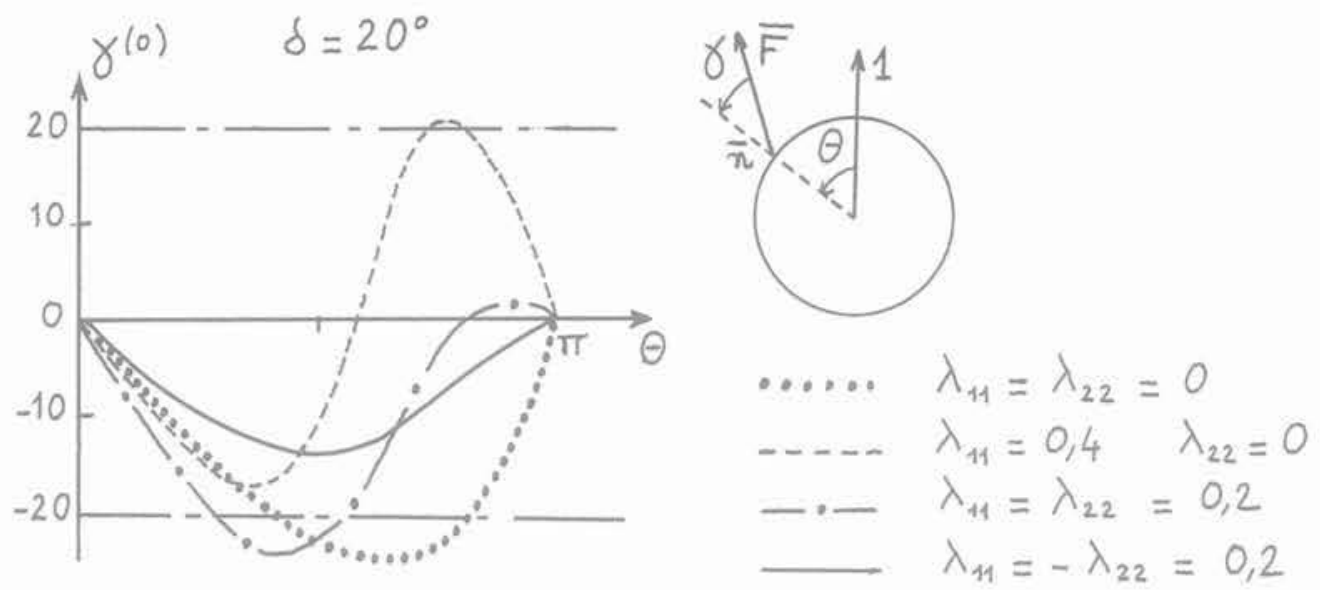

Fig. 5. - Distribution des orientations $\gamma$ des valeurs moyennes des forces de contact.

$\sigma_{\Delta}{ }^{(t)}$ est la part de $\sigma_{\Delta}$ due uniquement aux composantes tangentielles des forces de contact.

Pour une sollicitation sans rotation du repère principal $\left(\lambda_{12}=\lambda_{21}=0\right)$, (5) et (3) permettent de définir $\sigma_{\Delta}(\mathbb{N})$ en fonction de $\sigma_{\Delta}$

$\sigma_{1}^{(N)}=\sigma_{1}\left(\frac{3}{4}+\frac{\lambda_{11}}{4}\right)+\sigma_{11}\left(\frac{1}{4}-\frac{\lambda_{22}}{4}\right)$

Soit $s=\frac{\left|\sigma_{1}-\sigma_{\| 1}\right|}{\sigma_{1}+\sigma_{\| I}}=\mid \frac{\sigma_{1}^{(\mathrm{N})}-\sigma_{\| 1}^{(\mathrm{N})}}{\sigma_{1}+\sigma_{11}}$

$+\frac{\sigma_{1}^{(t)}-\sigma_{11}^{(t)}}{\sigma_{1}+\sigma_{11}}|=| s^{(n)}+s^{(t)} \mid$

s caractérise l'angle de frottement interne mobilisé,

(7) peut s'écrire aussi sous la forme:

$$
s^{(n)}=\left|\frac{1}{2} s+\frac{\lambda_{m}}{2} s+\frac{\lambda_{d}}{2}\right|
$$

avec $\lambda_{m}=\left(\lambda_{11}+\lambda_{22}\right) / 2$ et $\lambda_{d}=\left(\lambda_{11}-\lambda_{22}\right) / 2$

Les relations (7) et (8) montrent que l'évolution des variables d'état $\lambda_{\alpha \beta}$ traduit l'adaptation de la structure de façon à ce que le déviateur soit supporté pour une plus grande part par les composantes normales des forces de contact qui sont au niveau de chaque contact les éléments stabilisants.

On notera dans (8) que $\delta s^{(n)} / \delta \lambda_{m}=s / 2$ dépend du signe de $s$ et que $\delta s^{(n)} / \delta \lambda_{d}=\frac{1}{2}$ ne dépend pas $d u$ signe de $s$.

$\lambda_{\mathrm{m}}$ caractérise un mécanisme isotrope d'évolution de l'état et $\lambda_{\mathrm{d}}$ un mécanisme anisotrope.

En effet pour $\lambda_{\mathrm{d}}>0, \lambda_{\mathrm{m}}=0 \quad \mathrm{~s}^{(\mathrm{n})}>\left|\frac{1}{2} \mathrm{~s}\right|$

si $s>0$ et $s^{(n)}<\left|\frac{1}{2} s\right|$ si $s<0$
On notera de plus que $\delta s^{(n)} / \delta \lambda_{m}<\delta s^{(n)} / \delta \lambda_{d}$ pour toute valeur de s.

Donc $\lambda_{\mathrm{d}}$ correspond au mécanisme d'évolution optimum quel que soit $s$.

On peut donc considérer que, en première approximation, l'évolution de l'état est commandée par la seule variable d'état $\lambda_{\mathrm{d}}=\lambda_{11}-\lambda_{22}$ (Pour une sollicitation irrotationnelle).

Un programme de simulation numérique [1] nous a permis de montrer que $\lambda_{d}$ est compris entre - 0,35 et $+0,35$.

On notera que l'égalité $s^{(n)}=s$ correspond au cas où le déviateur est supporté uniquement par des forces normales. Par exemple pour un état défini par $\lambda_{\mathrm{d}}=0,3$ on peut calculer un angle de frottement interne mobilisable égal à $17,5^{\circ}$ sans frottement intergranulaire $(\mu=0)$.

Ces résultats sont en accord avec les travaux de THORNTON [14] qui en utilisant le programme numérique de Cundall [7] a montré que dans un milieu granulaire, ce sont essentiellement les forces normales qui supportent le déviateur (fig. 6).

Donc dans un milieu granulaire l'angle de frottement mobilisable est lié d'une part au frottement intergranulaire mais aussi et surtout à l'évolution de l'état du milieu $\left(\lambda_{d}\right)$ permettant aux composantes normales de supporter une part importante du déviateur.

\subsection{Critère de glissement}

La relation (1) traduit la condition de frottement au contact $\mathrm{k}$.

Par sommation sur tous les contacts définis pour une même orientation $\theta$, les valeurs moyennes des forces de contact doivent satisfaire à une condition semblable $|\overline{T(\theta)}| \leqslant \overline{N(\theta)} \mu$ ou encore $|P(\vec{n}) \overline{T(\vec{n})}| \leqslant P(\vec{n}) \overline{N(\vec{n})} \mu$ 

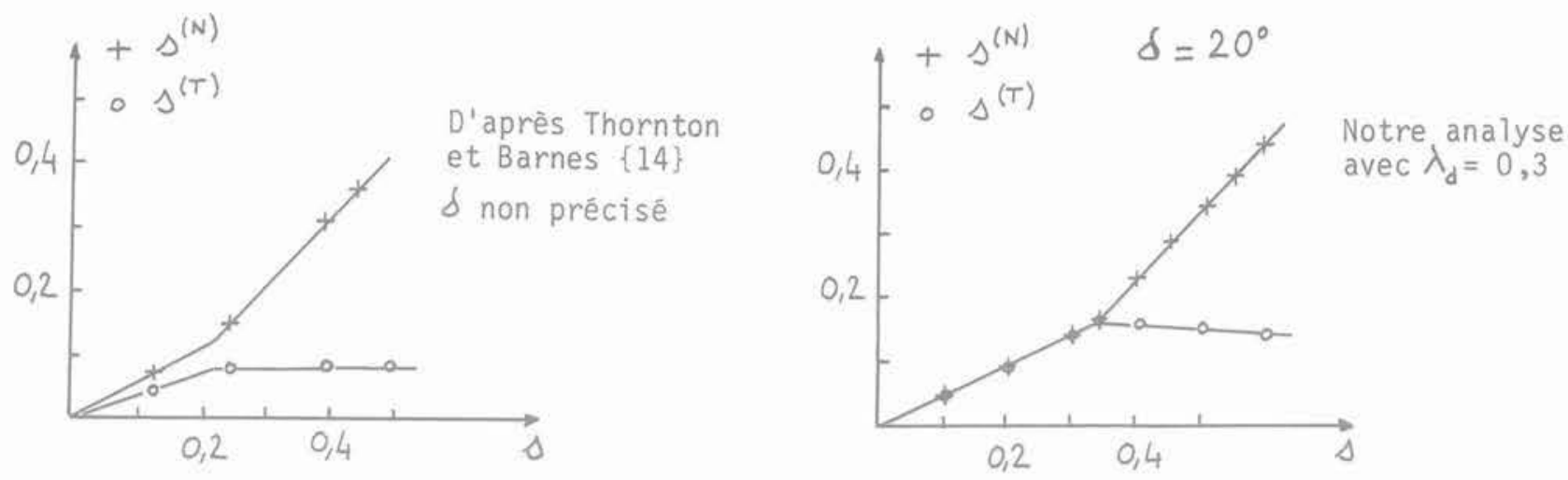

Fig. 6. - Contribution des composantes normales et tangentielles des forces de contact à l'angle de frottement mobilisé s.

On notera que si (9) est une condition nêcessaire pour que l'on soit dans un état possible, elle n'est pas a priori suffisante mais c'est la seule que nous pouvons écrire car nous ne disposons que d'informations sur les valeurs moyennes.

Pour aller plus loin, il faudrait raffiner la description statistique (voir \& 4).

Pour expliciter le critère (9), nous nous plaçons dans le repère principal des contraintes.

$\underline{P(\vec{n}) \tilde{N}}$ et $P(\vec{n}) T$ peuvent être calculées à partir de $P(\vec{n})$ $\overline{F_{\Delta}(\bar{n})}$ qui elles-mêmes peuvent être exprimées $(5)$ à partir des fonctions $f_{\Delta}(\vec{n})$.

La relation (9) s'écrit alors:

$$
\begin{aligned}
& \operatorname{Sup}_{\theta}\left\{\frac{\left(n_{1}-n_{2} \mu\right) n_{2} f_{2}}{\left(n_{1} \mu+n_{2}\right) n_{1} \tilde{f}_{1}}\right\} \\
& \leqslant \frac{\sigma_{1}}{\sigma_{2}} \\
& \leqslant \operatorname{Inf}_{\theta}\left\{\frac{\left.\left(n_{1}+n_{2} \mu\right) n_{2} f_{2}\right)}{\left(n_{2}-n_{1} \mu\right) n_{1} f_{1}}\right\}
\end{aligned}
$$

avec: $\mathrm{f}_{1}=\pi f_{1} / 2 \mathrm{n}_{1}$

$$
\mathrm{f}_{2}=\pi \mathrm{f}_{2} / 2 \mathrm{n}_{2}
$$

Le terme $\mathrm{f}_{2} / \mathrm{f}_{1}$ dépend de l'état du milieu et caractérise l'effet de mémoire dans le matériau.

Dans l'état isotrope de référence, on a $\mathrm{f}_{1}=\mathrm{f}_{2}=1$, les inégalités (10) caractérisent alors un critère de type "Mohr-Coulomb» d'angle de frottement interne égal à $\delta(\operatorname{tg} \delta=\mu)$.

$$
\operatorname{tg}^{2}(\pi / 4-\delta / 2) \leqslant \sigma_{1} / \sigma_{2} \leqslant \operatorname{tg}^{2}(\pi / 4+\delta / 2)
$$

Les orientations critiques sont:

$$
\left\{\begin{aligned}
\theta=\pi / 4+\delta / 2 & \text { pour } \sigma_{1}>\sigma_{2} \\
\theta=\pi / 4-\delta / 2 & \text { pour } \sigma_{1}<\sigma_{2}
\end{aligned}\right.
$$

Il est difficile d'écrire le critère en fonction des variables internes. Cependant on peut en donner une expression approchée pour $\lambda_{\mathrm{m}}=0$, en supposant que les orientations critiques varient peu avec $\lambda_{\mathrm{d}}$ :

$$
\begin{aligned}
& \operatorname{tg}^{2}\left(\frac{\pi}{4}-\frac{\delta}{2}\right) \frac{1+\lambda_{d}(1+2 \sin \delta)}{1+\lambda_{d}(-1+2 \sin \delta)} \leqslant \frac{\sigma_{1}}{\sigma_{2}} \\
& \leqslant \operatorname{tg}^{2}\left(\frac{\pi}{4}+\frac{\delta}{2}\right) \frac{1+\lambda_{d}(1-2 \sin \delta)}{1-\lambda_{d}(1+2 \sin \delta)}
\end{aligned}
$$

L'écriture de ce critère montre de nouveau que l'angle de frottement mobilisable dans un milieu granulaire est lié au frottement intergranulaire $\left(\operatorname{tg}^{2}(\pi / 4 \pm \delta / 2)\right)$ et à l'état du milieu (restructuration caractérisée par $\lambda_{\mathrm{d}}$ )

Le critère (12) peut aussi s'écrire sous la forme:

$$
\begin{array}{ll}
\sigma_{1}>\sigma_{2} & \frac{\sigma_{1}-\sigma_{2}}{\sigma_{1}+\sigma_{2}} \leqslant \frac{\sin \delta+\lambda_{\mathrm{d}} \cos 2 \delta}{1-\lambda_{\mathrm{d}} \sin \delta} \\
\sigma_{1}<\sigma_{2} & \frac{\sigma_{2}-\sigma_{1}}{\sigma_{1}+\sigma_{2}} \leqslant \frac{\sin \delta-\lambda_{\mathrm{d}} \cos 2 \delta}{1+\lambda_{\mathrm{d}} \sin \delta}
\end{array}
$$

Le critère $(12,13)$ permet de définir dans un plan $p, q$ (fig. 7) les courbes limitant le domaine « sans glissement». Ce sont des droites issues de l'origine et paramétrées par la variable d'état $\lambda_{\mathrm{d}}$.

Si nous nous plaçons dans le cadre d'une analyse élastoplastique, ces droites sont les représentations des fonctions de charge attachées au mécanisme d'écrouissage paramétré par $\lambda_{\mathrm{d}}$, ce mécanisme d'écrouissage est de type "cinématique» (fig. 8).

\subsection{Règle d'écoulement}

Pour définir la règle d'écoulement associée à ces fonctions de charge, nous écrivons que le travail plastique est égal au travail des forces de glissement:

$\sigma_{\alpha \beta} \mathrm{d} \varepsilon_{\alpha \beta}^{p}=\sum_{k=1}^{N} \vec{T}^{k} \overrightarrow{d g}^{k}=\int_{0}^{\pi} \overline{T(\vec{n}) d g(\vec{n})} d \theta$

$\overrightarrow{\mathrm{dg}}^{\mathrm{k}}$ : glissement relatif au contact $\mathrm{k}\left(\overrightarrow{\mathrm{T}}^{\mathrm{k}} \mathrm{dg}^{\mathrm{k}}>0\right)$ 


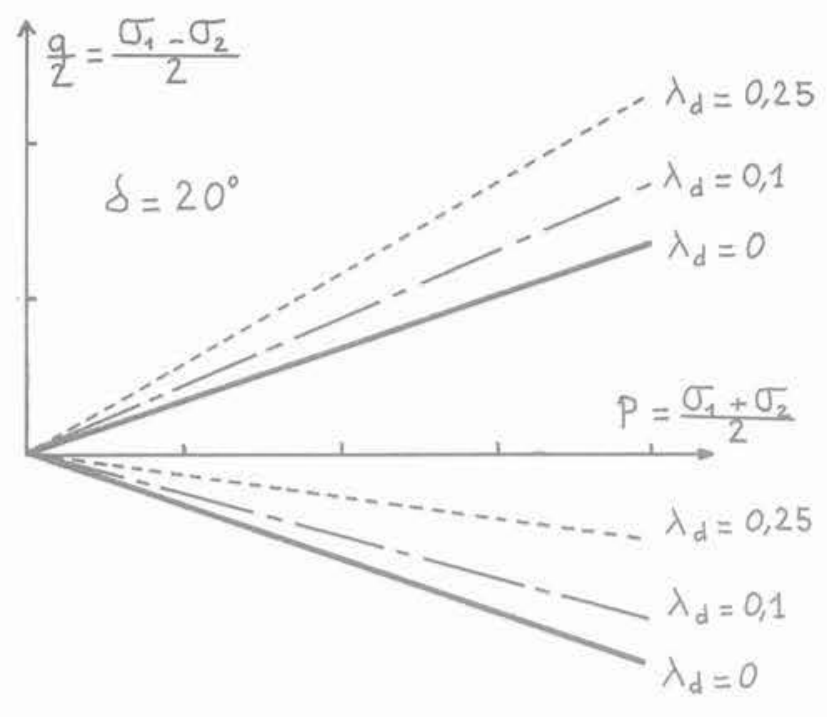

Fig. 7. - Surfaces de charge associées au mécanisme d'écrouissage $n^{\circ} 1$.

Soit:

$\mathrm{d} \tilde{\mathrm{g}}(\overrightarrow{\mathrm{n}})=\frac{\overline{T(\vec{n}) \mathrm{dg}(\overrightarrow{\mathrm{n}})}}{T(\vec{n})}$

On remarquera que $d \tilde{g}(\vec{n})$ est du signe de $T(\vec{n})$.

La relation (14) s'écrit dans le repère principal:

$\sigma_{1} d \varepsilon_{1}+\sigma_{\mathrm{II}} \mathrm{d} \varepsilon_{\mathrm{II}}$

$=\frac{1}{D} \int_{0}^{\pi}\left[-\sigma_{1} f_{I}(\vec{n}) n_{2}+\sigma_{11} f_{\| l}(\vec{n}) n_{1}\right] d \tilde{g}(\vec{n}) d \theta$

En identifiant en $\sigma_{1}$ et $\sigma_{\mathrm{II}}$ on obtient:

$$
\begin{array}{ll}
d \varepsilon_{1}=\frac{-1}{D} & \int_{0}^{\pi} f_{1}(\vec{n}) n_{2} d \tilde{g}(\vec{n}) \mathrm{d} \theta \\
d \varepsilon_{I I}=\frac{1}{D} & \int_{0}^{\pi} f_{I I}(\vec{n}) n_{1} d \tilde{g}(\vec{n}) \mathrm{d} \theta
\end{array}
$$

Ou encore en explicitant $f_{\mathrm{I}}$ et $\mathrm{f}_{\mathrm{II}}$ :

$d \varepsilon_{1}=-\frac{2}{\pi D} \int_{0}^{\pi}\left[1+\lambda_{d}(2 \cos 2 \theta-1)\right]$

$n_{1} n_{2} d \tilde{g}(\vec{n}) d \theta$

Pour toute valeur de $\theta$ :

$n_{1} n_{2} d g \tilde{(n)}$ est du signe de $n_{1} n_{2} T \overrightarrow{(\vec{n})}$ qui est lui-même du signe de $\left(\sigma_{2}-\sigma_{1}\right)$

Donc:

$$
\begin{gathered}
\mathrm{G}=(2 / \pi \mathrm{D}) \int_{0}^{\pi} n_{1} n_{2} d \tilde{g}(\vec{n}) \mathrm{d} \theta \text { est du signe de }\left(\sigma_{2}-\sigma_{1}\right) \\
(2 / \pi \mathrm{D}) \int_{0}^{\pi} 2 \cos 2 \theta \mathrm{n}_{1} \mathrm{n}_{2} \mathrm{dg}(\overrightarrow{\mathrm{n}}) \mathrm{d} \theta=\alpha \mathrm{G}
\end{gathered}
$$

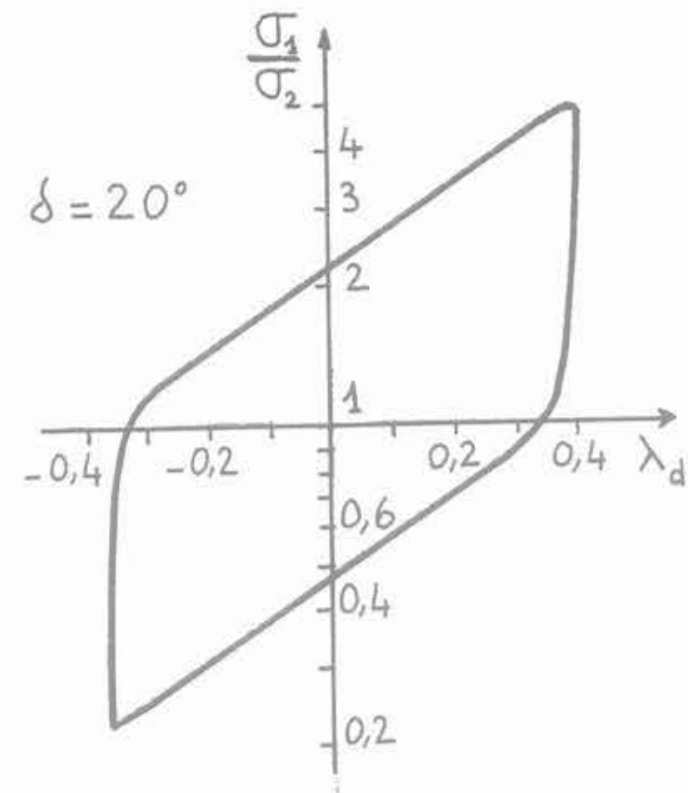

Fig. 8. - Critère de glissement paramétré par $\lambda_{d}$ (mécanisme d'écrouissage $n^{\circ} 1$ ).

On peut montrer que $-2<\alpha<+2$, dépend de la distribution des glissements dans la structure $(\mathrm{d} \tilde{g}(\tilde{n}))$.

La règle d'écoulement s'écrit alors:

$$
\begin{aligned}
& \mathrm{d} \varepsilon_{1}=-\mathrm{G}\left[1+\lambda_{\mathrm{d}}(-1+\alpha)\right] \\
& \mathrm{d} \varepsilon_{\mathrm{II}}=\mathrm{G}\left[1+\lambda_{\mathrm{d}}(1+\alpha)\right]
\end{aligned}
$$

Le potentiel plastique associé à (16) s'écrit:

$$
\left.\frac{\sigma_{1}}{\sigma_{2}}=\frac{1+\lambda_{d}(1+\alpha}{1+\lambda_{d}(-1+\alpha)}\right)
$$

On notera que la variation de volume défini par - (d $\varepsilon_{1}$ $\left.+\mathrm{d} \varepsilon_{I I}\right)$ ne dépend pas de $\alpha$, pour une sollicitation monotone, elle est toujours positive ou nulle:

$$
-\left(\mathrm{d} \varepsilon_{\mathrm{I}}+\mathrm{d} \varepsilon_{11}\right)=-2 \mathrm{G} \lambda_{\mathrm{d}}
$$

Si l'on fait l'hypothèse que la majorité des glissements se produit pour les orientations critiques (11) du critère (12) on peut alors exprimer $\alpha$ :

$$
\begin{array}{ll}
\text { pour } \sigma_{1}>\sigma_{2}: & \alpha=-2 \sin \delta \\
\text { pour } \sigma_{1}<\sigma_{2}: & \alpha=2 \sin \delta
\end{array}
$$

Dans ce cas la règle d'écoulement (16) et le potentiel plastique (17) sont parfaitement définis.

Dans cette hypothèse, la règle d'écoulement (16) et le critère (12) permettent d'écrire pour une sollicitation monotone en compression:

$$
\frac{\sigma_{1}}{\sigma_{\| 1}}=-\operatorname{tg}^{2}\left(\frac{\mathrm{II}}{4}+\frac{\delta}{2}\right) \frac{\mathrm{d} \varepsilon_{\| 1}}{\mathrm{~d} \varepsilon_{\mathrm{I}}}
$$


De même en extension:

$$
\frac{\sigma_{1}}{\sigma_{\mathrm{II}}}=-\operatorname{tg}^{2}\left(\frac{\mathrm{II}}{4}-\frac{\delta}{2}\right) \frac{\mathrm{d} \varepsilon_{\mathrm{ll}}}{\mathrm{d} \varepsilon_{\mathrm{I}}}
$$

Ces relations sont identiques aux relations contraintesdilatances qui ont été proposées par ROWE [11].

On notera que ces relations ont été obtenues moyennant une hypothèse simplificatrice sur le critère et sur la règle d'écoulement.

\section{DESCRIPTION STATISTIQUE AU $2^{e}$ ORDRE (MÉCANISME D'ÉCROUISSAGE $\left.\mathrm{N}^{\circ} 2\right)$}

Les distributions des variables aléatoires analysées dans le précédent chapitre $\left(\mathrm{P}(\overrightarrow{\mathrm{n}}) \quad \mathrm{F}_{\alpha}^{\mathrm{k}}\right)$ ont été décrites uniquement par leurs valeurs moyennes $\mathrm{P}(\overrightarrow{\mathrm{n}}) \hat{\mathrm{F}}_{\alpha}(\vec{n})$.

Nous nous proposons d'analyser ici la description à l'ordre supérieur en prenant, de plus, en compte les écarts types de ces distributions qui seront notés $\mathrm{P}(\bar{n}) \mathrm{s}_{\alpha \text {. }}$ En outre, nous supposons que ces distributions sont symétriques et limitées par les valeurs $P(\vec{n})$ $\left[\tilde{F}_{\alpha} \pm 2 s_{\alpha}\right]$ (Fig. 9).

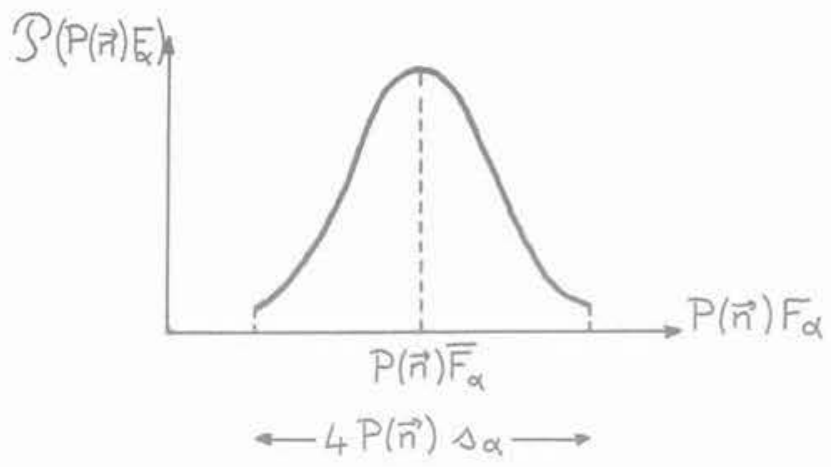

Fig. 9. - Distribution des variables $P(\vec{n}) F_{\alpha}$.

\subsection{Analyse et discrêtisation de l'écart type}

La relation (5) du paragraphe précédent exprime que la valeur moyenne des $F_{\alpha}^{k}$ est proportionnelle à la contrainte appliquée $\sigma_{\alpha \text {. }}$

Si l'on étend cette hypothèse à chacune des valeurs $\mathrm{F}_{\alpha}^{\mathrm{k}}$, le produit $\mathrm{P}(\overrightarrow{\mathrm{n}}) \mathrm{s}_{\alpha}(\mathrm{n})$ peut s'écrire sous la forme:

$P(\vec{n}) s_{\alpha}(\vec{n})=\frac{1}{N D} \sigma_{\alpha} v_{\alpha}(\vec{n})$

$v_{\alpha}(\vec{n})$ caractérise l'état du milieu, cette fonction joue un rôle analogue à la fonction $\mathrm{f}_{\alpha}$ utilisée pour la description au $1^{\text {er }}$ ordre (5), elle est indépendante de $\sigma_{\alpha}$
De même que pour la fonction $f_{\alpha}$ nous proposons de discréditer la fonction $v_{\alpha}(n)$ sous la forme:

$$
\begin{gathered}
v_{\alpha}(\vec{n})=V_{\alpha}-v_{\alpha \beta} n_{\beta}-V_{\alpha \beta \gamma} n_{\beta} n_{\gamma} \\
v_{\alpha}(\vec{n})=v_{\alpha}(-\vec{n}) \text { donc } V_{\alpha \beta}=0
\end{gathered}
$$

Pour une sollicitation à directions principales fixes le milieu conserve les symétries par rapport aux axes principaux 1 et 2 donc:

$v_{\alpha}(\theta)=v_{\alpha}(-\theta)$ et $v_{\alpha}(\theta)=v_{\alpha}(\pi-\theta)$

Donc $V_{112}=V_{212}=0$.

Si l'on pose pour simplifier l'écriture:

$$
V_{\alpha 1}=V_{\alpha 11} \text { et } V_{\alpha 2}=V_{\alpha 22}
$$

$V_{\alpha}$ s'écrit alors:

$$
v_{\alpha}(\vec{n})=V_{\alpha}-V_{\alpha \beta} n_{\beta}^{2}
$$

Pour une sollicitation isotrope $\left(\sigma_{\alpha}=\sigma\right)$, l'écart type des composantes normales $\left(\mathrm{s}_{\mathrm{N}}\right)$ et des composantes tangentielles $\left(\mathrm{s}_{\mathrm{T}}\right)$ des forces de contact s'écrit:

$$
\begin{aligned}
& s_{\mathrm{N}}^{2}=n_{1}^{2} s_{1}^{2}+n_{2}^{2} s_{2}^{2} \\
& s_{\mathrm{T}}^{2}=n_{2}^{2} s_{1}^{2}+n_{1}^{2} s_{2}^{2}
\end{aligned}
$$

Du fait de la symétrie du milieu $\mathrm{S}_{\mathrm{N}}$ et $\mathrm{s}_{\mathrm{T}}$ doivent être indépendants de $\vec{n}$ ce qui conduit aux conditions suivantes:

$V_{1}=V_{2}=V_{0} \quad$ et $V_{\alpha \beta}=0$

Donc à l'état vierge: $v(\vec{n})=V_{0}$

\subsection{Sollicitations déviatoires}

On peut montrer [1] que pour que le développement (20) soit unique, il faut fixer $V_{\alpha}$. Nous posons $V_{\alpha}=$ $V_{\alpha}, V_{\alpha}$ s'écrit alors:

$$
v_{\alpha}(\vec{n})=v_{0}-v_{\alpha \beta} n_{\beta}^{2}
$$

\subsubsection{Critère de glissement}

Si l'on prend en compte la largeur des distributions limitées à $2 \mathrm{~s}_{\alpha}$ le critère s'écrit:

$\operatorname{Sup}_{\theta}\left\{\frac{\left(n_{1}-n_{2} \mu\right)\left(n_{2} f_{2}+2 v_{2}\right)}{\left(n_{2}+n_{1} \mu\right)\left(n_{1} f_{1}-2 v_{1}\right)}\right\} \leqslant \frac{\sigma_{1}}{\sigma_{2}}$

$\leqslant \operatorname{Inf}_{\theta}\left\{\frac{\left(n_{1}+n_{2} \mu\right)\left(n_{2} f_{2}-2 v_{2}\right)}{\left(n_{2}-n_{1} \mu\right)\left(n_{1} f_{1}+2 v_{1}\right)}\right\}$

qui peut aussi s'écrire de façon approchée:

$$
\operatorname{tg}\left(\frac{\pi}{4}-\frac{\delta}{2}\right) \frac{f_{2} \sin \left(\frac{\pi}{4}-\frac{\delta}{2}\right)+2 v_{2}}{f_{1} \cos \left(\frac{\pi}{4}-\frac{\delta}{2}\right)-2 v_{1}}
$$




$$
\leqslant \frac{\sigma_{1}}{\sigma_{2}} \leqslant \operatorname{tg}\left(\frac{\pi}{4}+\frac{\delta}{2}\right) \frac{f_{2} \sin \left(\frac{\pi}{4}+\frac{\delta}{2}\right)-2 v_{2}}{f_{1} \cos \left(\frac{\pi}{4}+\frac{\delta}{2}\right)+2 v_{1}}
$$

Lorsqu'un milieu granulaire vierge est soumis à une sollicitation isotrope, on observe des déformations irréversibles, donc le critère (23) est satisfait pour:

$\sigma_{1}=\sigma_{2}, f_{1}=f_{2}=1$ et $v_{1}=v_{2}=V_{o}$

Cette condition permet de déterminer $V_{0}$.

$$
2 V_{0}=\sqrt{2} \sin (\delta / 2)
$$

Dans l'hypothèse oũ le mécanisme d'écrouissage caractérisé par $f_{\alpha}$ n'est pas activé, le critêre (23) est donc paramétré par les quatre paramètres définissant $v_{\alpha}: V_{\alpha \beta}$

Pour $\sigma_{1}>\sigma_{2} \quad\left|\partial v_{1} / \partial V_{12}\right|>\left|\partial v_{1} / \partial V_{11}\right|$

et $\left|\partial v_{2} / \partial V_{22}\right|>\left|\partial v_{2} / \partial V_{21}\right|$

On supposera donc que $V_{11}=V_{21}=0$ et que l'état du milieu est décrit par $V_{12}$ et $V_{22}$. On peut montrer de plus que seul le parametre $\left(V_{12}+V_{22}\right) / 2$ est significatif alors que $\left(V_{12}-V_{22}\right) / 2$ n'a qu'une faible influence sur l'évolution du critère (22).

On posera donc $V_{12}=V_{22}=V_{2}$ seul paramètre à évoluer pour $\sigma_{1}>\sigma_{2}$.

Pour $\sigma_{1}<\sigma_{2}$ le seul paramètre significatif de l'évolution du milieu est $V_{11}=V_{21}=V_{1}$.

Donc ce mécanisme d'écrouissage est paramétré par les deux variables $V_{\alpha}$ telles que:

$v_{1}(\vec{n})=v_{2}(\vec{n})=v(\vec{n})=v_{o}-v_{\alpha} n_{\alpha}^{2}$

Les fonctions de charges associées à ce mécanisme d'écrouissage sont des droites issues de l'origine (fig. 10).

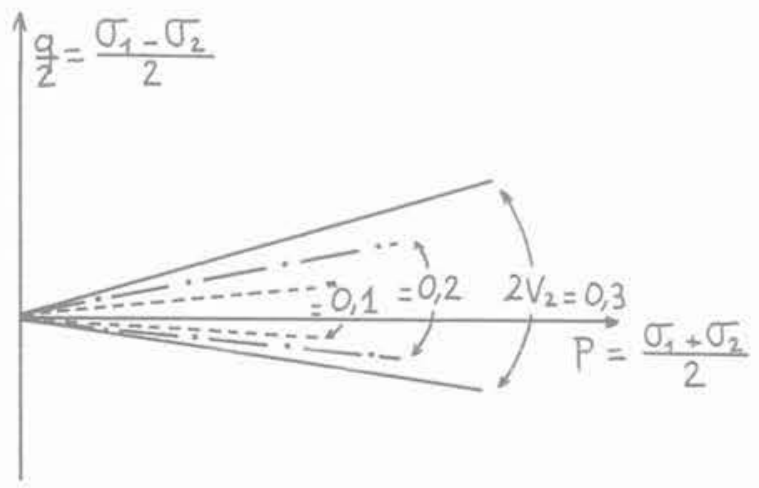

Fig. 10. - Fonctions de charge associées au mécanisme d'écrouissage $n^{\circ} 2$.
On peut voir sur la figure 11 que le mécanisme d'écrouissage paramétré par $V_{\alpha}$ est de type: (isotrope + cinématique).

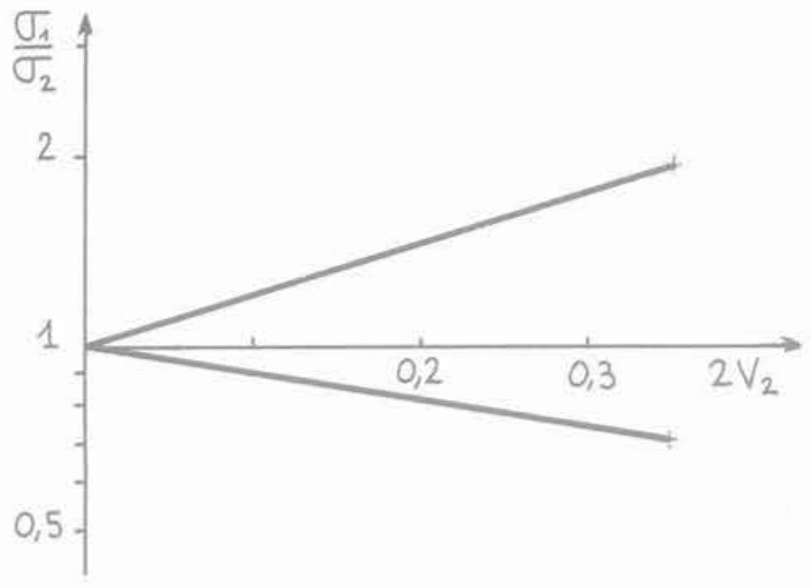

Fig. 11. - Critère de glissement paramétré par $V_{2}$ pour une sollicitation monotone croissante $\left(\sigma_{7}>\sigma_{2}\right)-$ Mécanisme d'écrouissage $n^{\circ} 2$.

\subsubsection{Règle d'écoulement}

Il est possible de définir la règle d'écoulement associée à ces fonctions de charge de la même façon que nous l'avons fait en 3.5., mais en posant:

$\mathrm{dg}(\overrightarrow{\mathrm{n}})=\frac{\mathrm{T}(\overrightarrow{\mathrm{n}}) \mathrm{dg}(\overrightarrow{\mathrm{n}})}{\mathrm{T}(\overrightarrow{\mathrm{n}}) \pm 2 \mathrm{~s}_{\mathrm{T}}(\overrightarrow{\mathrm{n}})}$

$(+$ si $T(\vec{n})>0 \quad-$ si $T(\vec{n})<0)$

On obtient ainsi:

$d \varepsilon_{l}=\frac{-1}{D} \int_{0}^{\pi}\left(f_{l}(\vec{n}) \pm 2 v\right) n_{2} d \tilde{g}(\vec{n}) d \theta$
$d \varepsilon_{\| l}=\frac{1}{D} \int_{0}^{\pi}\left(f_{\|}(\vec{n}) \pm 2 v\right) n_{1} d \tilde{g}(\vec{n}) d \theta$

Dans cette formule ainsi que dans les formules suivantes, le signe inférieur concerne $\sigma_{1}>\sigma_{2}$; le signe supérieur concerne $\sigma_{1}<\sigma_{2}$.

Dans le cas où le premier mécanisme n'est pas actif: $n_{I I} f_{1}=n_{1} f_{l l}$ et donc:

$$
-\left(d \varepsilon_{1}+d \varepsilon_{11}\right)= \pm \frac{2 v}{D}
$$

$\left[\int_{0}^{\pi} n_{1} d \tilde{g}(\vec{n}) d \theta+\int_{0}^{\pi} n_{2} d \tilde{g}(\vec{n}) d \theta\right]$

Pour une sollicitation sans rotation des directions principales dḡ $(\theta)$ est symétrique par rapport aux deux axes principaux de sollicitation, donc:

$$
\int_{0}^{\pi} n_{2} d \tilde{g}(\vec{n}) d \theta=0
$$

donc: $-\left(d \varepsilon_{1}+d \varepsilon_{n i}\right)= \pm \frac{2 v}{D} \int_{0}^{\pi} n_{1} d \tilde{g}(\vec{n}) d \theta$ 
$\int_{0}^{\pi} n_{1} d \tilde{g}(\vec{n}) d \theta$ est du signe de $\left(\sigma_{2}-\sigma_{1}\right)$ donc pour $v \neq 0$ la variation de volume: $-\left(d \varepsilon_{1}+d \varepsilon_{n !}\right)$ sera nécessairement négative (pour $\sigma_{1}>\sigma_{2}$ et pour $\sigma_{1}<$ $\left.\sigma_{2}\right)$.

Donc l'évolution du second mécanisme d'écrouissage paramétré par $V_{\alpha}$ se traduit nécessairement par une diminution de volume.

$\mathrm{Si}$ on fait l'hypothèse que la majorité des glissements se produit pour les orientations critiques (11) on obtient de même que pour le mécanisme précédent:

$$
\begin{gathered}
\frac{\sigma_{1}}{\sigma_{2}}=-\operatorname{tg}^{2}\left(\frac{\pi}{4} \pm \frac{\delta}{2}\right) \frac{\mathrm{d} \varepsilon_{\mathrm{ll}}}{\mathrm{d} \varepsilon_{1}} \\
\left.1+\text { pour } \sigma_{1}>\sigma_{2} \quad-\text { pour } \sigma_{1}<\sigma_{2}\right)
\end{gathered}
$$

Donc ces relations semblables aux relations contraintesdilatance de ROWE sont valables en première approximation aussi bien pour le deuxième mécanisme d'écrouissage que pour le premier.

\subsection{Sollicitation à déviateur constant}

L'analyse présentée ici fait apparaître des critères de glissement défini par le rapport $\sigma_{1} / \sigma_{2}$. Dans cette hypothèse, toute sollicitation telle que $\sigma_{1} / \sigma_{2}=$ cte ne provoquera pas de déformations irréversibles.

Ceci n'est urai qu'en première approximation, en particulier une sollicitation isotrope croissante $\left(\sigma_{1} / \sigma_{2}=1\right)$ se traduit par de petites déformations irréversibles.

Si l'on veut traduire ce phénomène, il faut modifier la relation (19) qui traduit l'hypothèse de proportionnalité des $F_{\alpha}^{k}$ à $\sigma_{\alpha}$. En effet, du fait des déformations au contact, le nombre de contacts (et donc $\mathrm{P}(\overrightarrow{\mathrm{n}})$ ) évolue légèrement avec la sollicitation. Ces déformations n'étant pas proportionnelles à $\sigma_{\alpha}$, la relation (19) n'est certainement acceptable qu'en première approximation.

Nous proposons donc de substituer à la relation (19) la nouvelle relation:

$\mathrm{P}(\overrightarrow{\mathrm{n}}) \mathrm{s}_{\alpha}(\overrightarrow{\mathrm{n}})=\frac{2}{\mathrm{ND}} \sigma_{\alpha} \vee \frac{\sigma_{\alpha}{ }^{\mathrm{a}}}{\mathrm{p}_{0}{ }^{\mathrm{a}}}=\frac{2}{\mathrm{ND}} \vee \frac{\sigma_{\alpha}{ }^{(1+\mathrm{a})}}{\mathrm{p}_{0}{ }^{a}}$

L'exposant a est un nombre $>0$ à déterminer, $\mathrm{p}_{0}$ égal à la pression atmosphérique permet d'assurer l'homogénéité de la formule (27).

L'analyse présentée en 4.2 . et 4.3 . peut être développée avec cette nouvelle hypothèse.

En particulier la nouvelle valeur de $V_{0}$ associée à une sollicitation isotrope $(\sigma)$ peut être définie:

$$
2 \mathrm{~V}_{\mathrm{o}}=\sqrt{2} \frac{\mathrm{p}_{\mathrm{o}}^{\mathrm{a}}}{\sigma^{\mathrm{a}}} \sin \frac{\delta}{2}
$$

On voit que lors d'une sollicitation isotrope croissante, $\mathrm{V}_{0}$ doit diminuer ce qui se traduira par une évolution de l'état du milieu donc des déformations irréversibles, ce qui correspond bien à la réalité expérimentale.
Les surfaces de charges associées au deuxième mécanisme ne sont plus des droites issues de l'origine mais présentent une légère courbure (qui dépend de la valeur de l'exposant a) (fig. 12).

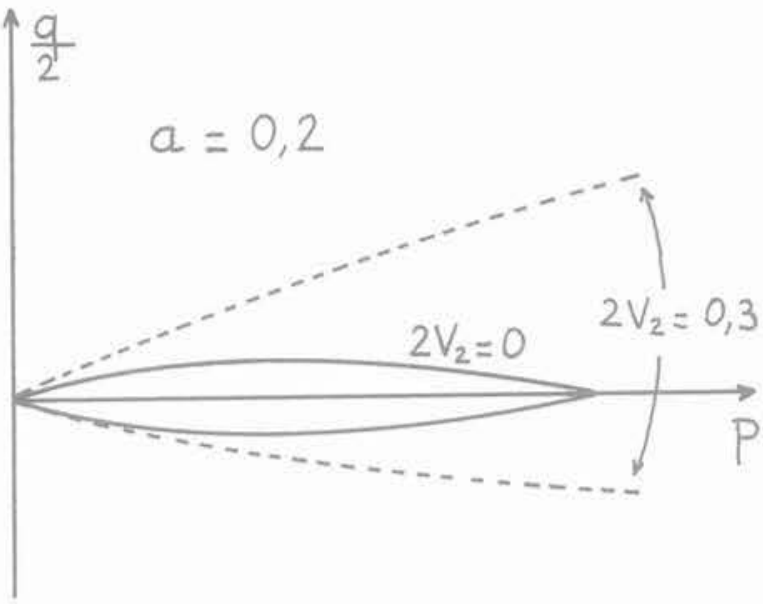

Fig. 12. - Fonctions de charge associées au mécanisme d'écrouissage $n^{\circ} 2$.

\section{MODĖLE PROPOSÉ DANS UNE APPROCHE ÉLASTOPLASTIQUE - CONFRONTATION AVEC L'EXPÉRIENCE}

\subsection{Modèle proposé}

L'analyse microstructurale présentée précédemment conduit, dans le cadre d'une analyse élastoplastique, à un modèle à deux mécanismes d'écrouissage (fig. 13):

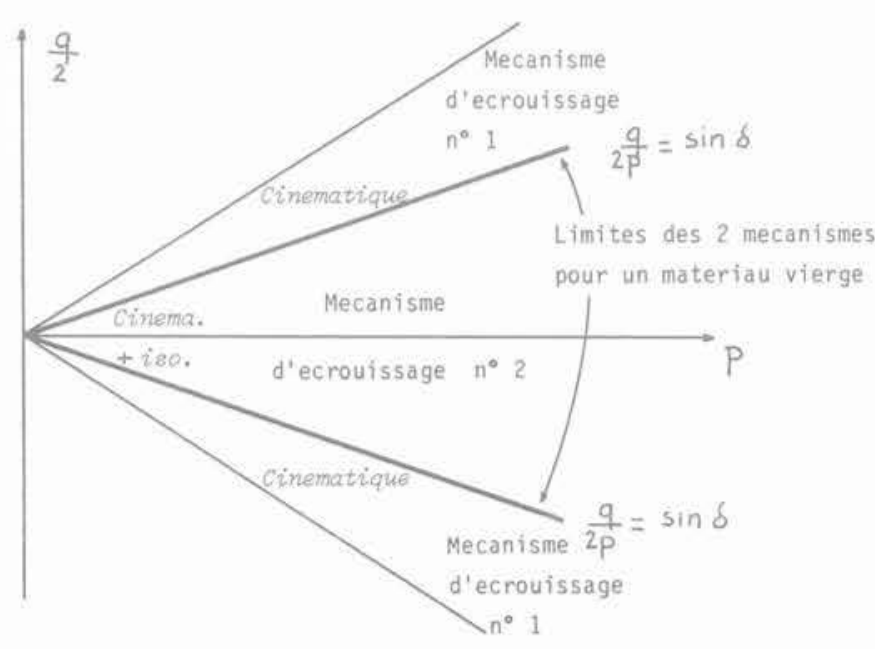

Fig. 13. - Domaines d'activation des mécanismes d'écrouissage 1 et 2 pour un matériau vierge. 
- Le mécanisme noté $n^{\circ} 1$, présenté au $\S 3$ traduit l'évolution des valeurs moyennes orientées des forces de contact.

Pour un milieu bidimensionnel et pour des sollicitations irrotationnelles, ce mécanisme est paramétré par un scalaire $\left(\lambda_{d}\right)$.

Ce mécanisme est de type cinématique. Pour une première sollicitation monotone, ce mécanisme est nécessairement activé pour $\sigma_{1} / \sigma_{2} \leqslant \operatorname{tg}^{2}(\pi / 4-\delta / 2)$ ou pour $\sigma_{1} / \sigma_{2} \geqslant \operatorname{tg}^{2}(\pi / 4+\delta / 2)$.

La fonction de charge et le potentiel plastique associés à ce mécanisme sont représentés dans un plan p,q par des droites issues de l'origine (fig. 7).

Pour une sollicitation monotone, lorsque ce mécanisme est activé, la variation de volume est positive (dilatance). Pour des sollicitations complexes (cycliques) la variation de volume peut être positive ou négative selon le cas.

- Le mécanisme noté $n^{\circ} 2$ et présenté au $\$ 4$ traduit l'évolution de la largeur des distributions orientées des forces de contact.

Pour un milieu bidimensionnel et pour des sollicitations irrotationnelles, ce mécanisme est paramétré par deux scalaires $\left(\mathrm{V}_{\alpha}\right)$.

Ce mécanisme est de type (isotrope + cinématique). Pour une première sollicitation monotone, ce mécanisme est activé dès le début de la sollicitation.

Ce mécanisme ne peut être activé seul que pour:

$$
\operatorname{tg}^{2}(\pi / 4-\delta / 2)<\sigma_{1} / \sigma_{2}<\operatorname{tg}^{2}(\pi / 4+\delta / 2)
$$

La fonction de charge et le potentiel plastique associés à ce mécanisme sont représentés dans un plan p,q par des lignes légèrement incurvées issues de l'origine (fig. 12).

Lorsque ce mécanisme est activé seul, la variation de volume est négative (contractance).

Lorsque l'un ou l'autre de ces deux mécanismes sont activés les déformations irréversibles satisfont en première approximation, à la relation contrainte-dilatance de ROWE.

Pour une première sollicitation monotone la figure 13 montre les domaines d'activation des différents mécanismes.

Après sollicitation, les limites de ces domaines évoluent, il y a création d'un domaine "élastique» (fig. 14).

On peut penser que dans la réalité, le passage de l'activation de l'un des mécanismes d'écrouissage à l'autre se fera progressivement au voisinage des limites précédemment définies.

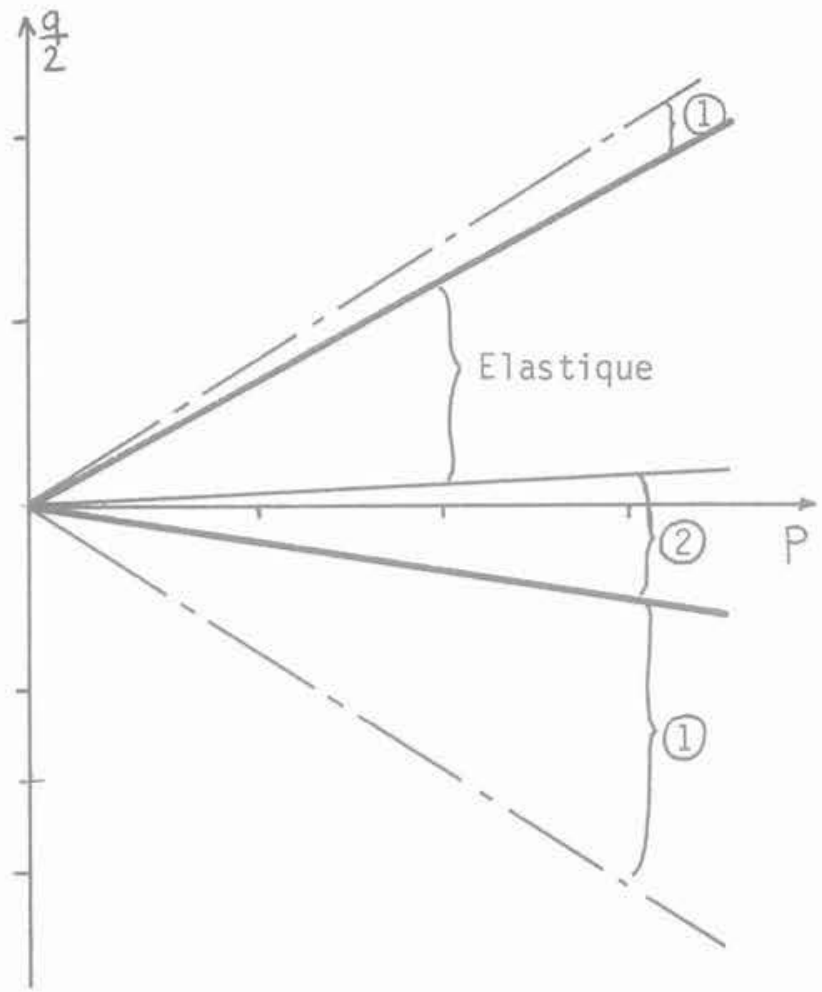

Fig. 14. - Domaine d'activation des mécanismes d'écrouissage 1 et 2 pour un matériau déjà écroui en compression $\left(\sigma_{1}>\sigma_{2}\right)$.

\subsection{Confrontation avec la réalité expérimentale}

\section{- Sollicitation isotrope}

L'hypothèse (27) traduit l'existence de déformations irréversibles sous sollicitation isotrope, qui vont être commandées par l'évolution de V.

$$
\frac{\mathrm{dV}_{0}}{\mathrm{d \sigma}}=-\frac{\mathrm{a}}{\sqrt{2}} \mathrm{p}_{0}^{\mathrm{a}} \sin \delta \frac{1}{\sigma^{(\mathrm{a}+1)}}
$$

La relation (28) est compatible avec la forme de la courbe experimentale (fig. 15),

Moyennant certaines hypothèses supplémentaires l'exposant a pourrait être identifié à partir de la courbe expérimentale: $\sigma=f\left(\Delta V^{P} / V_{0}\right)$.

\section{- Première sollicitation déviatoire monotone}

L'expérience montre l'existence d'un domaine où le matériau est contractant et d'un domaine où le matériau est dilatant. HABIB et LUONG $[8,10]$ ont montré que ces deux domaines sont limités dans le plan p,q par une droite dite droite caractéristique qui ne dépend pas de la densité du matériau (fig. 16).

Ces résultats expérimentaux sont en accord avec notre analyse. Le domaine subcaractéristique est le domaine 


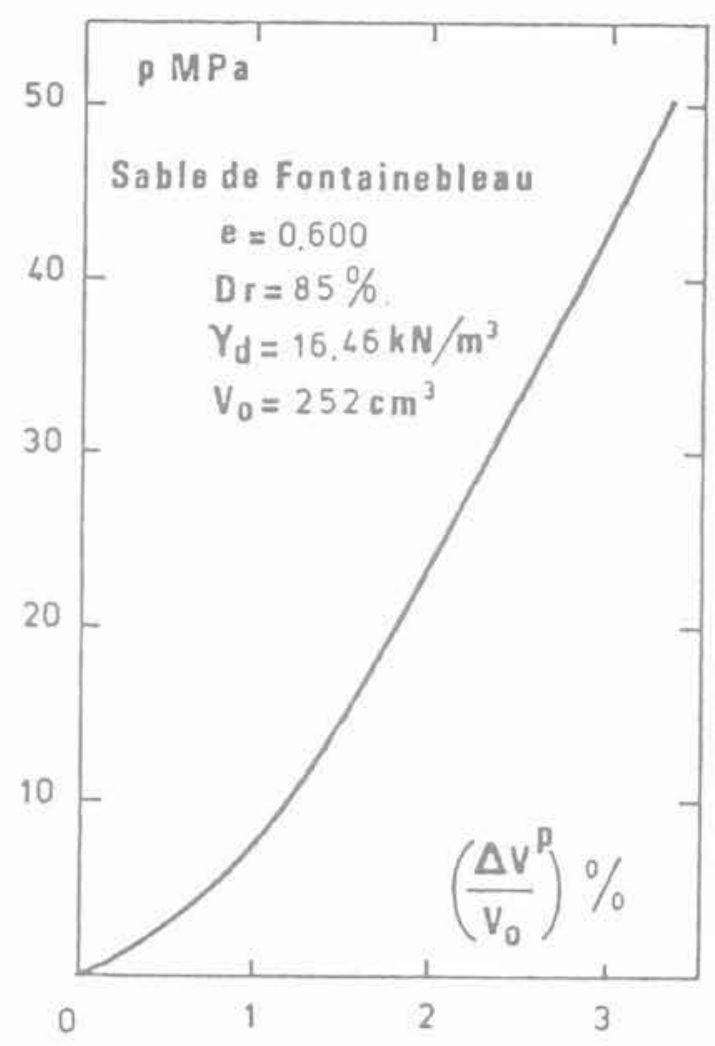

Fig. 15. - Déformation volumique irréversible sous contrainte isotrope.

(d'après Luong [10]).

d'activation du mécanisme d'écrouissage $n^{\circ} 2$ (contractance), le domaine surcaractéristique est le domaine d'activation du mécanisme d'écrouissage $n^{\circ} 1$ (dilatance). La limite entre les deux domaines est indépendante de la densité du milieu (fig. 13).

Il est possible de déterminer expérimentalement les fonctions de charge. Les représentations de ces fonctions de charge sont des lignes légèrement incurvées issues de lorigine (fig. 17).

Ces résultats expérimentaux sont en accord avec notre modèle (fig. 7,12 ).

Les expériences de compression-extension représentées sur la figure 18 permettent de définir le type d'écrouissage. Pour une faible valeur du déviateur, le mécanisme d'écrouissage semble être de type (isotrope + cinématique), et pour un déviateur plus élevé, de type cinématique, ce qui est parfaitement compatible avec le modèle proposé.

\section{- Sollicitations cycliques}

Lors de sollicitations cycliques de compressionextension de grande amplitude, les variations de volume montrent une tendance générale au compactage, même pour des échantillons denses [13] (fig. 19). $\mathrm{Ce}$ phénomène est correctement traduit par le modèle proposé. Lors de la $1^{\text {re }}$ sollicitation en compression, les mécanismes 1 et 2 sont activés, $\lambda_{d}$ évolue $\left(\lambda_{d}>0\right)$,

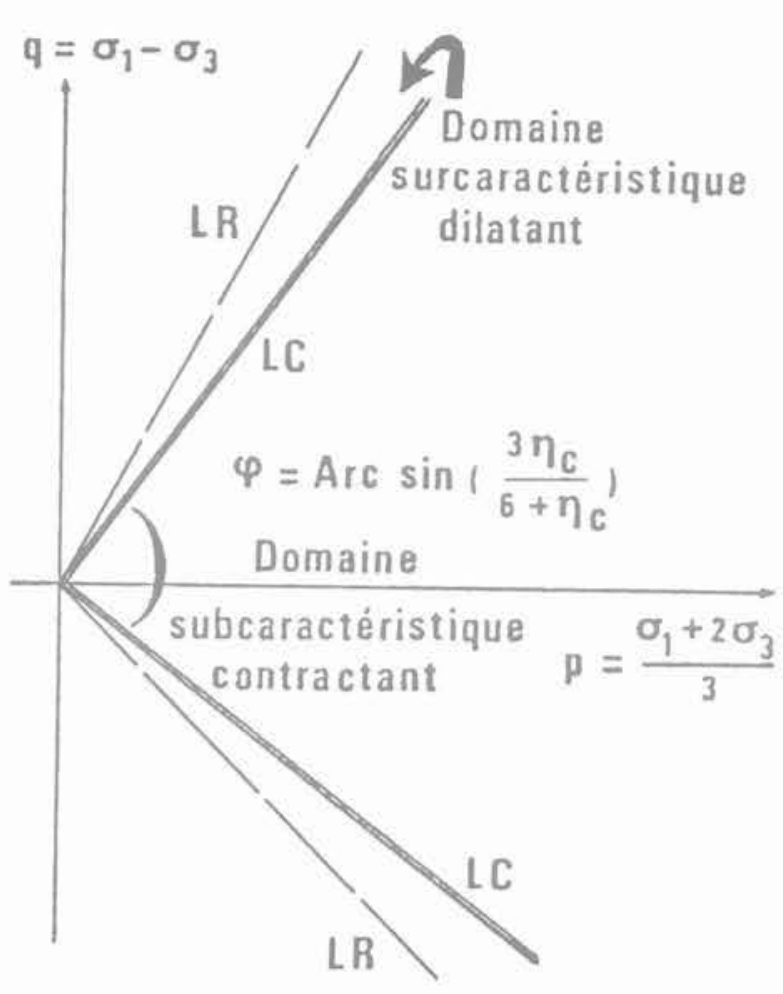

Fig. 16. - Critère caractéristique. (d'après Luong [10]).

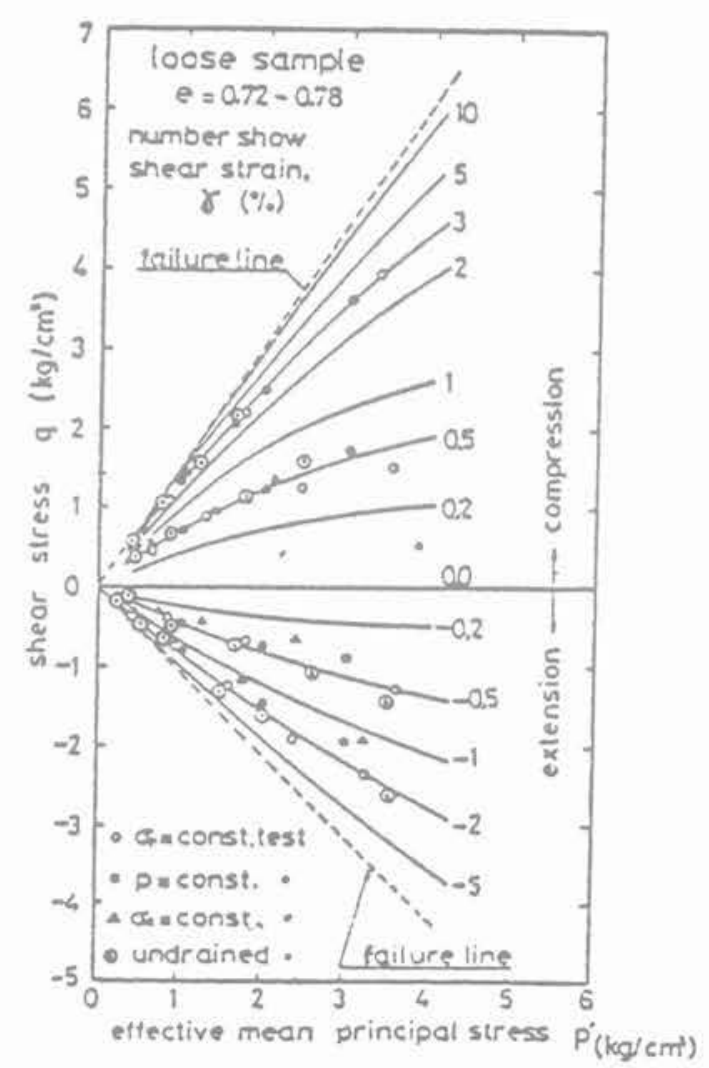

Fig. 17. - Recherche d'une surface de charge par cisaillement drainé.

(d'après Tatsuoka et Ishihara [12]). 

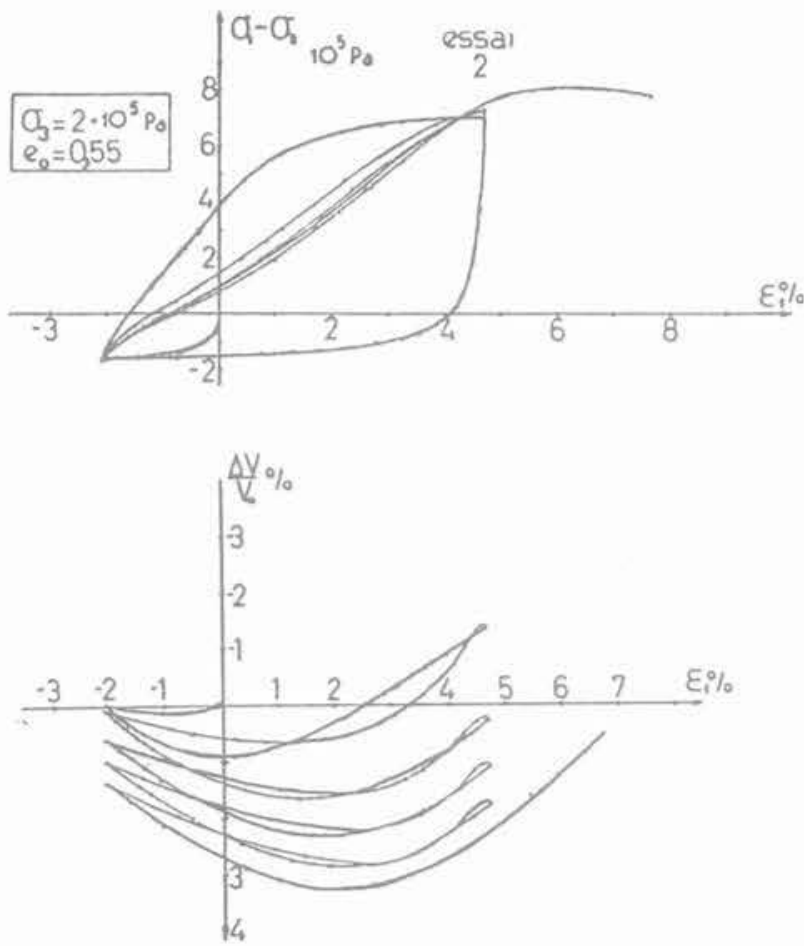

Fig. 18. - Essais triaxiaux

de compression-extension. Sable $-\gamma_{d}=15,4 \mathrm{kN} / \mathrm{m}^{3}$ $\sigma_{3}=20 \mathrm{kPa}$.

quand la sollicitation est inversée le mécanisme 2 est activé (contractance), puis le mécanisme 1 (en extension), la variation de volume associée est alors égale à :

$$
-\left(d \varepsilon_{1}+d \varepsilon_{15}\right)=-2 G \lambda_{d}
$$

$\mathrm{G}$ est du signe de $\sigma_{2}-\sigma_{1}($ donc $>0)$.

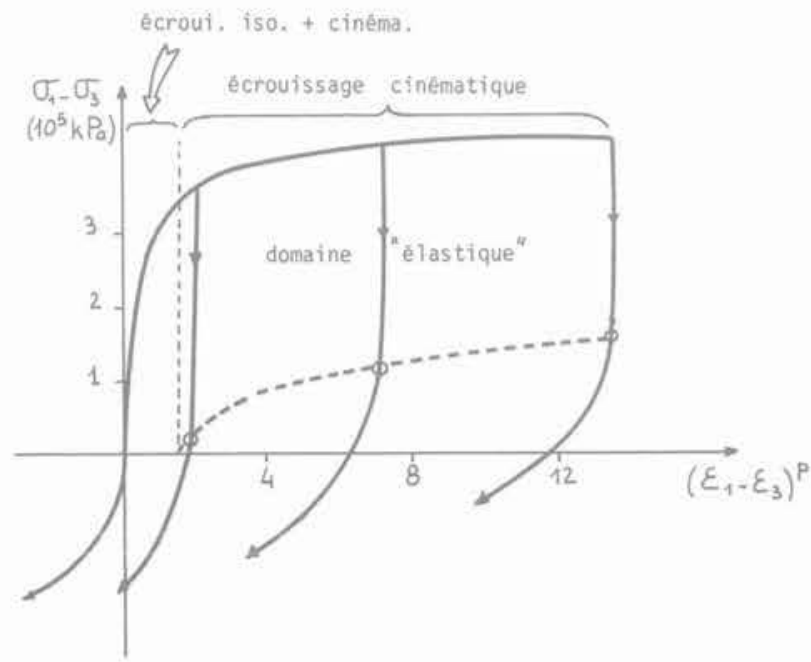

Fig. 19. - Cycles de grande amplitude entre deux valeurs de déformation axiale (Sable dense). (d'après Thanopoulos [13]). $\lambda_{\mathrm{d}}$ est $>0$ au début de l'activation du mécanisme $\mathrm{n}^{\circ} 1$, donc la variation de volume est alors négative. Si la sollicitation en extension se poursuit, $\lambda_{\mathrm{d}}$ évolue et devient négatif (légère augmentation de volume). Après une nouvelle inversion du sens de sollicitation, il se produit de nouveau une contractance suivi d'une légère dilatance en fin de cycle et le processus se reproduit ainsi pour chaque cycle (fig. 19).

\section{CONCLUSION}

L'approche microstructurale présentée ici a permis de définir les mécanismes d'écrouissage d'un modẻle élastoplastique à partir de la réalité physique fondamentale.

Le modèle proposé semble traduire l'essentiel des comportements observés expérimentalement.

L'analyse présentée a été limitée aux cas de sollicitations irrotationnelles. L'analyse de sollicitations avec rotation des directions principales ne présente aucune difficulté théorique mais augmente le nombre de paramètres d'écrouissage, elle fera l'objet d'un prochain développement.

\section{BIBLIOGRAPHIE}

[1] CAMBOU B, (1982). - - Orientational distributions of contact forces as memory parameters in a granular material». C.R du colloque IUTAM «Deformation and failure of granular Material». Delft.

[2] CAMBOU B. (1984). - - Mécanique des matériaux granulaires, l'approche microstructurale». Cours de l'Ecole d'Hiver des Géomatériaux. C.N.R.S. I.M.G. - Aussois.

[3] CAMBOU B. (1984). - *Microscopic aspects of hardening in granular materials. C.R. du Congrès International CHISA - Prague.

[4] CAMBOU B.; SIDOROFF F. (1983). - «Failure criteria for granular material based on statical Microstructural variables *. C.R. colloque C.N.R.S. Villard-de-Lans - Juin 1983.

[5] CAMBOU B. ; SIDOROFF F. (1984), - a Distributions orientées dans un milieu granulaire et leurs représentations ». C.R. Journées de Mécanique Aléatoire appliquée à la construction. Paris.

[6] CAMBOU B.; SIDOROFF F. (1985). - ${ }^{\alpha}$ Description de l'état d'un matériau granulaire par variables internes statiques à partir d'une approche discrète». Journal de Mécanique Théorique et Appliquée (à paraître).

[7] CUNDALL P.A.; STRACK O.D.L. (1979). - A discrete numerical model for granular assemblies». Géotechnique n²9 - pp. $47 / 65$.

[8] HABIB P. ; LUONG M.P (1978). - « Sols pulvérulents sous chargements cycliques». Séminaire Matériaux et Structures sous chargement cyclique, 28-29 sept. 1978, Ecole Polytechnique, Palaiseau. 
[9] LORET B. (1984). - «Application de la théorie des multimécanismes à l'étude du comportement des sols». Cours de l'Ecole d'Hiver des Géomatériaux - C.N.R.S. I.M.G. - Aussois.

[10] LUONG M.P. (1980). - «Phénomènes cycliques dans les sols pulvérulents». Revue Française de Géotechnique, n¹0, pp. 39/53.

[11] ROWE P.W. (1969). - - The relation between the shear strenght of sands in triaxial compression, plane strain and direct shear». Géotechnique 19 Vol. 1 - pp. $75 / 86$

[12] TATSUOKA F.; ISHIHARA K. (1974). - «Yiel- ding of sand in triaxial compression *. Soils and Foundations, 14, 2, pp. 63/76.

[13] THANOPOULOS 1. (1981). - « Contribution à l'étude du comportement cyclique des milieux pulvérulents». Thèse D.I. Grenoble.

[14] THORNTON C.; BARNES (1984). - "The relationship between stress and microstructure in particulate media». C.R. du Congrès International CHISA - Prague.

[15] WEBER J. (1966). - «Recherche concemant les contraintes intergranulaires dans les milieux pulvérulents». Bull. Liaison P. et C. $n^{\circ} 20-p$. 3.1/3.20, 
